\title{
Spontaneous-Regeneration of the Reconstructed Mandible in a Segmental Mandibulectomized Young Girl
}

\author{
Ikawa T', Shigeta $\mathrm{Y}^{1 *}$, Kihara T1, Ando $\mathrm{E}^{1}$, Harada \\ $N^{2}$, Hirai $S^{1}$, Shigemoto $S^{1}$, Nakaoka $K^{3}$, Hamada \\ $\mathbf{Y}^{3}$ and Ogawa $\mathbf{T}^{1}$ \\ ${ }^{1}$ Department of Fixed Prosthodontics, Tsurumi \\ University, J apan \\ ${ }^{2}$ Dental Technician Training Institute, Tsurumi \\ University, J apan \\ ${ }^{3}$ Department of Oral and Maxillofacial Surgery, Tsurumi \\ University, J apan \\ *Corresponding author: Shigeta Y, Department \\ of Fixed Prosthodontics, School of Dental Medicine, \\ Tsurumi University, Yokohama, J apan
}

Received: December 10, 2018; Accepted: J anuary 22, 2019; Published: January 29, 2019

\begin{abstract}
Spontaneous bone regeneration of the reconstructed mandible in children is rarely reported. Most of previous reports discussed the role of periosteum in regeneration of bone. Alternatively, other factors such as mechanical stress and muscle attachment have also been implicated in spontaneous bone regeneration. This paper presents spontaneous-regeneration of the reconstructed mandible in a young girl, undergone a segmental mandibulectomy, and discusses the factors associated with regeneration based on the results of image examinations and Finite Element Analysis (FEA). The patient was an 11 year-old girl diagnosed as ossifying fibroma in the mandible. A segmental mandibulectomy and immediate mandibular reconstruction using a Ti-mesh tray and particulate cancellous bone and marrow was planned. The spontaneousregeneration of the coronoid process was observed on the reconstructed mandible. On the CT slabs, the reattachment of the masseter and temporal muscle was confirmed. Before treatment, the transversal growth of her maxilla was asymmetrical. After the mandibular reconstruction and prosthodontic treatment, her maxilla grew back with bilateral symmetry. The occlusal loading on her mandible appeared symmetrically via an FEA. In addition, the stress was concentrated in the external oblique ridge, and the graft bone remodeled into the anatomical configuration. Through this case, it is suggested that the reattachment, the healthy growth of masticatory muscles, and the rehabilitation of oral function contributed to spontaneous bone regeneration and remodeling in the mandible, as well as the preservation of periosteum.
\end{abstract}

Keywords: Mandibular reconstruction; Self-regeneration; Finite element analysis

\section{Introduction}

Even in children, various oral and maxillofacial tumours develop. In 1997, Sato et al. [1] reported incidences and diagnosis of 250 oral and maxillofacial tumours in children. They reported that 232 patients $(93 \%)$ had benign tumours and 18 (7\%) were malignant. In some cases, the tumours disappear without treatment as the child is growing. On the other hand, depending on the type of tumor, surgical ablation may be needed.

Spontaneous bone regeneration of the reconstructed mandible in children is rarely reported in the previous literatures. In 2012, Ahmad et al. [2] presented a case with spontaneous regeneration following hemimandibulectomy, and discussed the theories pertaining to the mechanism, and source of the new bone formation in the light of previous literature. They focused on the role of periosteum in regeneration of bone. Other literature [3] has also described that the reservation of periosteum, coupled with the age of the patients, possible genetic factors, and infection may play important roles in rapid bone regeneration. Alternatively, other factors such as mechanical stress and muscle attachment have also been implicated in spontaneous bone regeneration.

In this paper, we present a case of spontaneous-regeneration of the reconstructed mandible, and discuss on the relationship between regeneration and muscle reattachment based on the findings

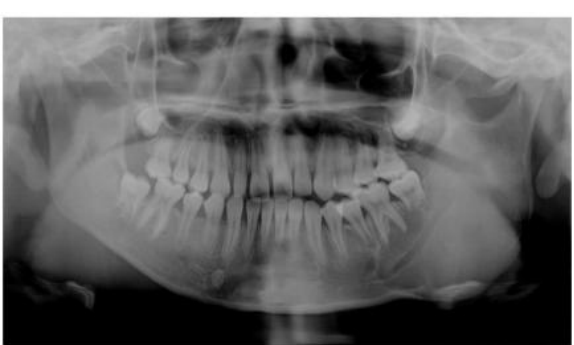

Figure 1: Initial panoramic image.

of Computer Tomography (CT). In addition, the influences of occlusal relation and oral function on the mandibular growth were investigated through the analysis of occlusal force and Finite Element Analysis (FEA).

\section{Case Presentation}

The patient was an 11 year-old girl. She complained of facial swelling and visited our hospital. As for 3 years history, she slowly had an enlargement of painless swelling on the left cheek. Visual inspection showed severe swelling from the left mandibular angle to the ramus region. Manual examination showed diffuse, non-tender, hard-texture swelling. An intraoral examination showed buccolingual expansion of the mandibular alveolus in the left molar region 

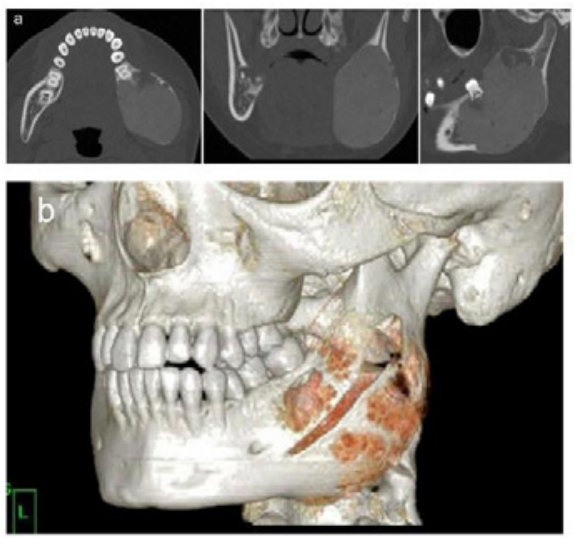

Figure 2: a) Initial Computer Tomography images. b) The expanded tumorlike lesion was observed on the three-dimensional (3D) CT image.

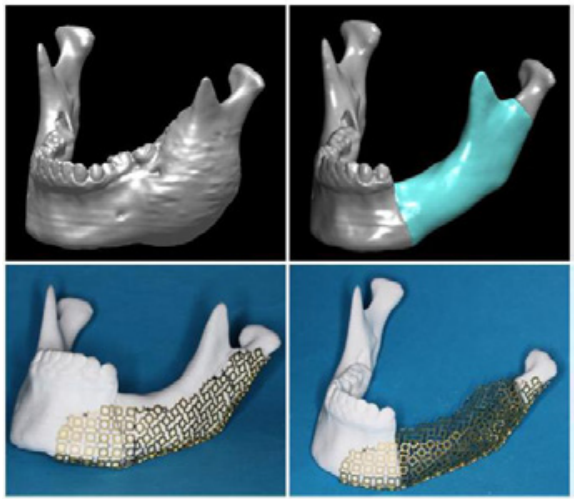

Figure 3: The surgical planning on the virtual model and the fabrication of Ti-mesh tray on the physical model of the mandible.
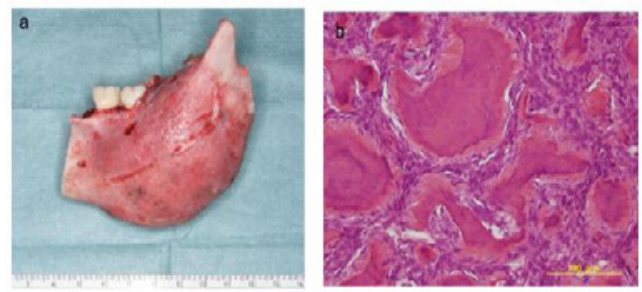

Figure 4: a) The surgical specimen. b) The histopathological image

with overlying of normal mucosa. Her left molars were vital and inclined to the lingual side. There was no neural disturbance.

On the initial panoramic examination there was a large oval radiolucent lesion from the left molar region to the ramus (Figure 1). CT examination showed the lesion extending anteriorly from the left second premolar to the coronoid process and notch posteriorly, sparing the condylar process (Figure 2). The biopsy confirmed the provisional diagnosis of an ossifying fibroma. We planned a segmental mandibulectomy of the left mandible from the second premolar to the mandibular notch, including the coronoid process, and immediate mandibular reconstruction using a custom-made Timesh tray and autogenous Particulate Cancerous Bone And Marrow (PCBM) harvested from posterior iliac crest.

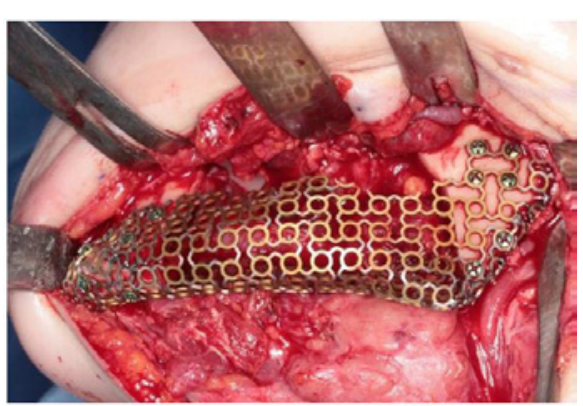

Figure 5: Setting of Ti-mesh tray to the simulation physical model.

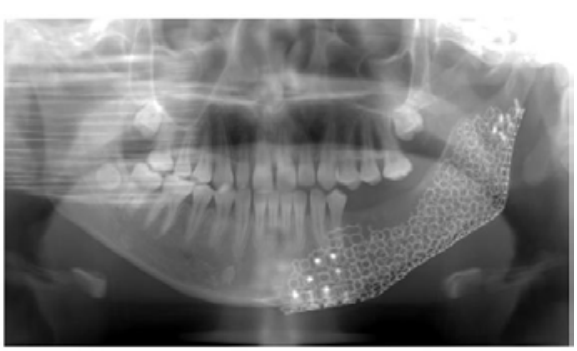

Figure 6: X-ray findings 1 year after surgical treatment.
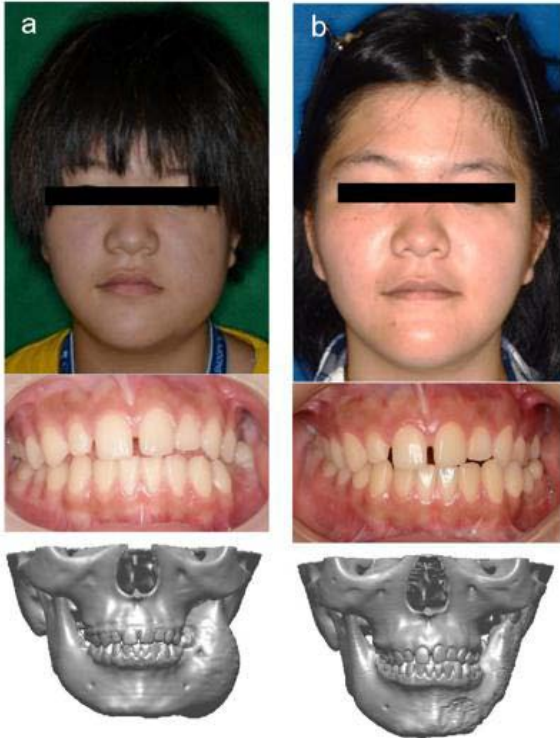

Figure 7: Facial, intraoral and X-ray image findings at pre- and posttreatment. a) pre-treatment. b) 5 years after.

Our treatment plan of attempting a mandibular reconstruction, incorporating a custom-processed Ti-mesh tray, was reviewed and approved by the Ethics Committee at Tsurumi University (Approval number:915). This study followed the Declaration of Helsinki on medical protocol. Informed consent was obtained from the patient and her parents.

In our previous report, we presented each step of the treatment procedures for computer-assisted mandibular reconstruction in detail [4-6]. The same procedures were applied for this case. Figure 3 shows each step of the surgical planning on the virtual and physical 
model, and the fabrication of the Ti-mesh tray. Figure 4 shows the surgical specimen and histopathological findings led to the diagnosis of ossifying fibroma. The Ti-mesh tray was set up according to the simulation (Figure 5). Two years after the surgery, there was no remarkable abnormal resorption of the reconstructed mandible. In addition, we were able to observe the formation of cortical bone, on the superior margin of the reconstructed mandible, and the occlusal reconstruction using dental implants were applied to the left molar region of her reconstructed mandible.

$\mathrm{X}$-ray and reconstructed CT images show a spontaneousregeneration of the coronoid process 1 year after mandibular reconstruction (Figure 6). Five years after the surgery, her body height increased $4 \mathrm{~cm}$ (from 160 to $164 \mathrm{~cm}$ ), and her weight increased $13 \mathrm{~kg}$ (from 51 to $64 \mathrm{~kg}$ ). Her facial symmetry and the stability of occlusal relation were maintained (Figure 7).

\section{Discussion}

An ossifying fibromas, which appear in pediatric patients $(<15$ years-old), is called juvenile ossifying fibroma [7]. It is thought that these tumors develop in the jaw from undifferentiated cells of the periodontal ligament in the premolar and molar regions [8]. As in our case, Espinosa et al. reported spontaneous-regeneration after juvenile ossifying fibroma resection [9]. However, their case was treated with the segmental resection of mandibular body, and the ramus was saved.

In this case, the spontaneous-regeneration of the coronoid process was observed on the reconstructed mandible. Figure 8 shows the superimposition of the virtual simulation data and the reconstructed CT data 5 years after the treatment. On the healthy side, the condyle and coronoid processes were growing with her systemic growth. On the treated side, the slightly growing condyle and the regeneration of the muscle insertion areas of masseter and temporal muscles were observed in the reconstructed mandible. Furthermore, the bone resorption was observed in the buccal side of left molar region, and then the left external oblique ridge was formed on the reconstructed mandible.

The previous papers have reported the crucial role of periosteum in osteogenesis, and revealed the regeneration of bone by progenitor cells of periosteum [10-14]. Therefore, the preservation of periosteum is an important consideration to spontaneous bone regeneration following segmental mandibulectomy. Because mechanical stress and muscle attachment are other factors implicated in spontaneous bone regeneration, the masseter and temporal muscles were observed on the post-treatment CT and MRI images (Figure 9). On the 4 slabs of CT and MRI data, the reattachments of the masseter and temporal muscle were confirmed. On slab D and d, it was confirmed that the regenerated coronoid process is surrounded by the temporal muscle. Furthermore, these muscles were growing as well as the healthy side. These findings support the relation between regeneration and muscle attachment. In 2017, Castellon et al. [15] reported the remodeling of costochondral graft after mandibular reconstruction. They presented the unusual remodeling of a nonvascularized costochondral graft in a segmental mandibulectomized pediatric patient. The condyle and coronoid process were regenerated on the graft. Consequently, they concluded that the reattachment of muscles and periosteum

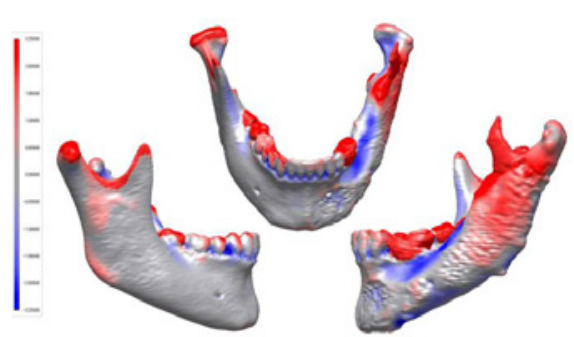

Figure 8: Superimposition image of the simulation and the CT data of posttreatment. Red: over $3 \mathrm{~mm}$ increase, Blue: over $3 \mathrm{~mm}$ decrease.

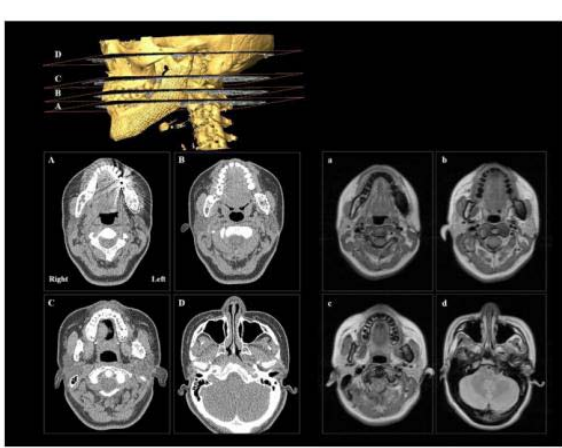

Figure 9: Observation of muscles on CT and MRI images. A-D: CT images, (a-d): MRI images corresponding to 4 slabs of CT.
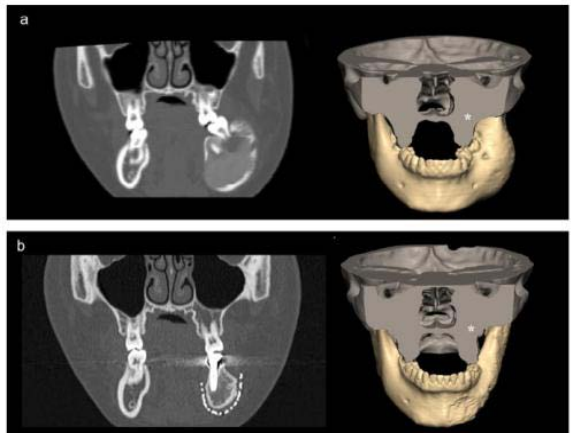

Figure 10: Images of frontal slabs and reconstructed CT data before and after treatment (a) pre-treatment, (b) post-treatment). ${ }^{*}$ Cranial model was clipped in the mesial of first molar.

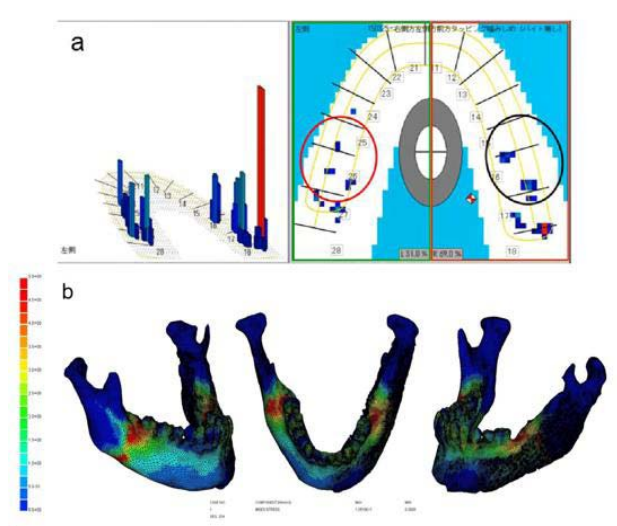

Figure 11: a) Occlusal relation via T-scan. b) Stress on the mandible via FEA 
regenerative capacity could explain the particular remodeling of the CCG in our patient. The phenomenon in our patient supports their theory.

In 1995, Kiliaridis [16] reviewed the influence of the masticatory muscle function on craniofacial growth. It was concluded that the increased loading of the jaws, due to masticatory muscle hyperfunction, may lead to increased sutural growth and bone apposition, resulting in an increased transversal growth of the maxilla and broader bone bases for the dental arches. Furthermore, an increase in the function of the masticatory muscles is associated with anterior growth rotation pattern of the mandible and with well-developed angular, coronoid, and condylar processes.

Figure 10 shows the images of the frontal slabs and 3D-reconstructed CT data. Before treatment, the left mandibular molars had transferred and inclined to the lingual side due to the growing tumor, and her left occlusal relation collapsed. In addition, her transversal growth of the maxilla was slightly asymmetrical. The phenomenon in our patient was supported by the above theory. After treatment, her maxillary bone, especially the supporting alveolar bone, grew to the infra-buccal side.

In addition, the occlusal relation was analyzed after treatment via T-scan, and the stress on the mandible during occlusion was evaluated via FEA. After the mandibular reconstruction and prosthodontic treatment with the dental implants, her occlusal relation and function was restored (Figure 11A). In addition, the occlusal loading on her mandible appeared symmetrically via a FEA (Figure 11B). Under the occlusal force, the stress was concentrated in the external oblique ridge. From these findings it was considered that the mechanical stress and the muscle attachment were also contributed to the regeneration and remodeling of the mandible.

\section{Conclusion}

Through this case, it is suggested that the reattachment, the healthy growth of masticatory muscles, and the rehabilitation of oral function contributed to spontaneous bone regeneration and remodeling in the mandible, as well as the preservation of periosteum.

\section{References}

1. Sato M, Tanaka N, Sato T, Amagasa T. Oral and maxillofacial tumours in children: a review. Br J Oral Maxillofac Surg. 1997; 35: 92-95.

2. Ahmad O, Omami G. Self-Regeneration of the Mandible Following Hemimandibulectomy for Ameloblastoma: A Case Report and Review of Literature. J Maxillofac Oral Surg. 2015; 14: 245-250.
3. Nwoku AL. Unusually rapid bone regeneration following mandibular resection. J Maxillofac Surg. 1980; 8: 309-315

4. Ikawa T, Shigeta Y, Hirabayashi R, Hirai S, Hirai K, Harada N, et al. Computer assisted mandibular reconstruction using a custom-made titan mesh tray and removable denture based on the top-down treatment technique. J Prosthodont Res. 2016; 60: 321-331.

5. Yamada H, Nakaoka K, Sonoyama T, Kumagai K, Ikawa T, Shigeta $Y$, et al. Clinical Usefulness of Mandibular Reconstruction Using Custom-Made Titanium Mesh Tray and Autogenous Particulate Cancellous Bone and Marrow Harvested From Tibia and/or Ilia. J Craniofac Sur. 2016; 27: 586-592.

6. Yamada H, Nakaoka K, Horiuchi T, Kumagai K, Ikawa T, Shigeta Y, et al. Mandibular reconstruction using custom-made titanium mesh tray and particulate cancellous bone and marrow harvested from bilateral posterior ilia. J Plast Surg Hand Surg. 2014; 48: 183-190.

7. Williams HK, Mangham C, Speight PM. Juvenile ossifying fibroma. An analysis of eight cases and a comparison with other fibro-osseous lesions. $J$ Oral Pathol Med. 2000; 29: 13-18.

8. Leimola-Virtanen R, Vabatalo K, Syrjanen S. Juvenile active ossifying fibroma of the mandible: a report of 2 cases. J Oral Maxillofac Surg. 2001; 59: 439-444.

9. Espinosa SA, Villanueva J, Hampel H, DDS, Reyes D. Spontaneous regeneration after juvenile ossifying fibroma resection: a case report. Oral Surgery, Oral Medicine, Oral Pathology, Oral Radiology, and Endodontology. 2006; 102: 32-35.

10. Mc Kibbin B. The biology of fracture healing in long bones. J Bone Joint Surg Br. 1978; 60: 150-162.

11. Ito Y, Fitzsimmons JS, Sanyal A. Localization of chondrocyte precursors in periosteum. Osteoarthritis Cartilage. 2001; 9: 215-223.

12. Li M, Amizuka N, Oda K. Histological evidence of the initial chondrogenesis and osteogenesis in the periosteum of a rib fractured model: implications of osteocyte involvement in periosteal chondrogenesis. Microsc Res Tech. 2004; 1 : 330-342.

13. Ogunlewe MO, Akinwande JA, Ladeinde AL, Adeyemo WL. Spontaneous regeneration of whole mandible after total mandibulectomy in a sickle cell patient. J Oral Maxillofac Surg. 2006; 64: 981-984.

14. Okoturo E, Ogunbanjo OV, Arotiba GT. Spontaneous Regeneration of the Mandible: An Institutional Audit of Regenerated Bone and Osteocompetent Periosteum. J Oral Maxillofac Surg. 2016; 74: 1660-1667.

15. Castellon L, Jerez D, Mayorga J, Fuenzalida C. Remodeling of Costochondral Graft after Mandibular Reconstruction. J Oral Maxillofac Surg. 2017; 75: 226(e1-e7).

16. Kiliaridis S. Masticatory muscle influence on craniofacial growth. Acta Odontol Scand. 1995; 53: 196-202.
Austin J Surg - Volume 6 Issue 3 - 2019

ISSN : 2381-9030 | www.austinpublishing group.com

Shigeta et al. (C) All rights are reserved
Citation: Ikawa T, Shigeta Y, Kihara T, Ando E, Harada N, Hirai S, et al. Spontaneous-Regeneration of the Reconstructed Mandible in a Segmental Mandibulectomized Young Girl. Austin J Surg. 2019; 6(3): 1164 